Itinéraires Itinéraires

Littérature, textes, cultures

2009-2 | 2009

Caraïbe et océan Indien

\title{
1947 : Jean-Luc Raharimanana et Thierry Bedard contre l'oubli
}

Entretien

\section{Guillaume Bridet}

\section{CpenEdition}

\section{Journals}

Édition électronique

URL : http://journals.openedition.org/itineraires/313

DOI : 10.4000/itineraires.313

ISSN : 2427-920X

Éditeur

Pléiade

\section{Édition imprimée}

Date de publication : 1 juillet 2009

Pagination : 147-165

ISBN : 978-2-296-09639-4

ISSN : $2100-1340$

\section{Référence électronique}

Guillaume Bridet, « 7947 : Jean-Luc Raharimanana et Thierry Bedard contre l'oubli », Itinéraires [En ligne], 2009-2 | 2009, mis en ligne le 04 juin 2014, consulté le 04 mai 2019. URL : http:// journals.openedition.org/itineraires/313 ; DOI : 10.4000/itineraires.313

\section{Ce document a été généré automatiquement le 4 mai 2019.}

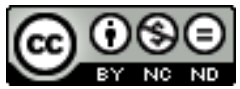

Itinéraires est mis à disposition selon les termes de la licence Creative Commons Attribution - Pas d'Utilisation Commerciale - Pas de Modification 4.0 International. 


\title{
1947 : Jean-Luc Raharimanana et Thierry Bedard contre l'oubli
}

\author{
Entretien
}

\section{Guillaume Bridet}

1 L'écrivain malgache Jean-Luc Raharimanana est l'auteur d'une œuvre qui, depuis le milieu des années 1990, prend de plus en plus d'ampleur par sa variété générique (théâtre, nouvelle, roman, essai), mais surtout par son ambition, puisqu'il en vient dans ses derniers textes à prendre ouvertement en charge l'histoire de son pays. Avec certaines nouvelles de Rêves sous le linceul (1998), mais surtout avec le roman Nour, 1947 (2001) et l'essai Madagascar, 1947 (2007), il s'arrête sur l'insurrection du peuple malgache qui eut lieu en 1947 et que l'armée française réprima dans le sang. Avec $Z a$ (2008), c'est cette fois l'histoire contemporaine de son pays, ressourcée aux mythes ancestraux de l'île de Madagascar, qu'il envisage dans une langue d'une rare et nouvelle inventivité.

2 Thierry Bedard est depuis maintenant près de vingt ans un metteur en scène exigeant, à la fois résolument politique et hostile à toute forme de consommation culturelle affadissante. Que ce soit dans ses mises en scène de textes de Michel Leiris (L'Afrique fantôme) en 1991-1992, de Danilo Kis (Encyclopédie des morts) en 1998-1999 ou de Reza Baraheni (Les Saisons en enfer du jeune Ayyâz) en 2002-2004, il scrute l'histoire du Xxe siècle et l'histoire contemporaine en portant l'attention vers ce qui dérange : les non-dits où se perçoit l'envers des sociétés démocratiques.

3 La rencontre de Jean-Luc Raharimanana et de Thierry Bedard donne lieu depuis maintenant près de deux ans à un travail commun autour de l'insurrection malgache de 1947 contre l'occupation française. Adaptée du livre Madagascar, 1947 et créée (non sans difficulté, on va le voir) en septembre 2008, la pièce intitulée 47 interroge de la manière la plus profonde l'histoire de la colonisation, les souffrances qu'elle a engendrées et qui restent tues.

4 L'entretien que l'un et l'autre ont eu la gentillesse de m'accorder s'est déroulé à Paris le 24 février 2009. 


\section{Des rencontres}

GUILLAUME BRIDET : Je suis heureux de m'entretenir avec chacun d'entre vous : avec Thierry Bedard, que les hasards de la vie m'ont fait connaître il y a longtemps et dont le travail de metteur en scène, même à distance, m'a toujours inspiré un certain respect ; avec Jean-Luc Raharimanana, puisque modestement je mets mes pas dans ceux de Jean-Louis Joubert qui, comme moi aujourd'hui, enseigna longtemps à Paris 13 et dont vous m'avez confié qu'il est le premier universitaire français à avoir écrit sur votre œuvre'. J'aimerais poser une première question à Thierry: comment as-tu découvert l'œuvre de Jean-Luc Raharimanana?

THIERRY BEDARD : Je crois que j'ai lu Lucarne il y a dix ans, quand le livre est sorti ${ }^{2}$. J'étais attentif à tout ce que publiait son éditeur, Le Serpent à plumes. J'ai lu très régulièrement tout ce qui est sorti au fil des années, quelquefois avec retard. J'ai lu en particulier les auteurs qui, comme Jean-Luc, ont travaillé sur le Rwanda.

\section{G. B. : Et votre rencontre à tous deux ?}

T. B. : La rencontre a eu lieu en 2007 , lorsque, pour un autre travail, je me suis rendu à Antananarivo. Je me suis retrouvé dans les quartiers les plus pauvres de la ville et j'avais emmené avec moi le dernier ouvrage en date de Jean-Luc, L'Arbre anthropophage ${ }^{3}$, que j'avais déjà lu, mais que j'ai relu là-bas. Je crois aussi que j'avais remmené les nouvelles de Lucarne. Et là, il y a eu une sorte d'évidence. L'œuvre de Jean-Luc m'est apparue nécessaire pour répondre - mais sans répondre - à tout le trouble que j'éprouvais lors de mon séjour dans ce pays et à Antananarivo. Ensuite, l'enchaînement est simple. Je suis engagé dans une sorte de cycle de travail, qui s'appelle « de l'étranger [s]». Ce cycle consiste à aller vers des auteurs de plusieurs continents et à leur commander un état du monde vu de l'endroit où ils vivent ou dont ils sont originaires. Dans ce cas précis, j'ai laissé de côté mon projet initial, pas très solide alors, et qui avançait un peu de travers, et j'ai passé commande à Jean-Luc d'un état du monde vu de... de... je ne sais où ! Sa réponse a été immédiate. C'est au même moment qu'a été publié Madagascar, 1947. Je connaissais alors très peu de chose sur l'insurrection malgache (de la même manière qu'on sait très peu de chose sur l'insurrection de Sétif en 1945). J'ai été profondément choqué d'apprendre le fond de cette histoire. À ce moment-là, il m'est apparu tout à fait évident que 1947 était un épisode historique intéressant à traiter entre un Français et un Malgache : traiter de cet effroi et de ses suites.

G. B. : Et vous, Jean-Luc Raharimanana, quand Thierry Bedard demande à vous rencontrer, vous connaissez déjà son travail?

JEAN-LUC RAHARIMANANA : Je n'avais jamais vu son travail, je le connaissais juste de nom, grâce à un ami, qui s'appelle Soeuf Elbadawi et qui l'avait rencontré à La Réunion. J'ai appris sur internet qu'il avait travaillé sur La Bibliothèque Censurée $e^{4}$. Très souvent, les metteurs en scène contemporains refusent l'engagement et la politique. Je me trouvais là au contraire face à un metteur en scène qui était sur ce terrain. Quand il m'a contacté, je suis allé le voir. Ce qui m'a tout de suite frappé, c'est qu'il était en train de rêver devant moi. Il avait un monde qu'il construisait. On voyait bien qu'il avait lu mes livres, mais ce n'était pas ce qui m'intéressait dans sa démarche. Son but n'était pas juste de faire un bel objet théâtral dans le cadre d'une carrière bien précise. Il avait quelque chose à construire et à dire. 
T. B. : À l'origine de notre rencontre, il y a donc une commande.

J.-L. R. : J'avais fini deux textes, Madagascar, 1947 et le roman Za. Za n'était pas encore publié et j'étais dans l'indécision. Est-ce que j'allais rester chez mon éditrice Joëlle Losfeld ou est-ce que j'allais partir ${ }^{5}$ ? Je savais dès le départ que $Z a$ était un vrai don fait aux théâtreux. La difficulté qu'avait l'éditrice pour le lire ne m'inquiétait pas. Je savais que, pour un metteur en scène et pour un comédien, $Z a$ serait quelque chose d'intéressant. Mais je venais d'achever ce livre-là et je ne savais pas comment m'en sortir. Je ne pouvais plus écrire comme avant et je me demandais comment faire à présent. Et Thierry me commande un texte. J'étais embarrassé et, en même temps, c'était une chance. Ce projet me forçait à trouver d'autres moyens, d'autres formes d'écriture, et surtout pas dans le genre du roman. Au bout de notre deuxième ou troisième rencontre, j'ai donné à Thierry le manuscrit de Za et Madagascar, 1947, qui venait de sortir. Et il est revenu deux jours plus tard en me disant qu'il allait adapter pour la scène l'un et l'autre livre.

G. B. : On va parler longuement de 47 , spectacle qui a déjà été monté. Mais qu'en est-il du spectacle qui devait être créé à partir de $Z a$ ?

T. B. : Il n'est pas à l'ordre du jour actuellement, parce que je n'ai pas trouvé l'argent pour le produire. Mais on m'a proposé de faire une lecture de Za. On a donc monté une esquisse de ce que pourrait être la pièce. Ce spectacle a été créé en décembre à Annecy, sous le titre d'Excuses et dires liminaires de $Z a^{6}$. C'est essentiellement une performance musicale, avec un acteur et un musicien malgache. L'ensemble dure quarante-cinq minutes. Nous éprouvions la nécessité de faire quand même quelque chose.

\section{De Nour, 1947 à Madagascar, 1947}

G. B. : Revenons à 47. J'aimerais d'abord vous interroger, Jean-Luc Raharimanana, sur le passage de Nour, 1947 à Madagascar, 1947 puis à 47. La reprise (répétition et variation) joue un rôle important dans votre œuvre. Dans le cas présent, vous abordez un même objet, les événements de 1947 à Madagascar, en multipliant les formes d'approche.

J.-L. R. : C'est une bonne question, c'est la question de mon écriture. En principe, Nour, 1947 aurait dû être mon premier livre. C'est devenu le troisième ou quatrième. J'avais fini en 1990 une pièce de théâtre Le Prophète et le Président J'avais aussi fini Lucarne. Je voulais m'attaquer à un roman. Mais j'étais sorti de Lucarne complètement à la ramasse. Lucarne est un premier livre écrit avec les tripes, dans un élan, je ne dirais pas suicidaire, mais au cours duquel je me suis privé de tout, sauf de mots. Je me suis retrouvé à l'hôpital et me suis demandé jusqu'où j'irais comme ça. Je voulais écrire un deuxième livre, mais est-ce que je n'allais pas y laisser ma peau ? J'avais vingt et un, vingt-deux ans. Commencer comme ça, ça n'était pas bon du tout. Il fallait que je réfléchisse sur les raisons qui me poussaient à écrire dans ces conditions. La conclusion à laquelle je suis parvenu, c'est que je devais réfléchir sur l'histoire malgache et sur la création, dans un sens très large, pas simplement création littéraire, mais aussi création d'une identité et création d'une nation. Au départ, l'insurrection malgache et la répression française de 1947 ne participaient pas de mon approche. M'importait surtout un conte, qui revient tout le temps dans mon œuvre, y compris dans $Z a$, un conte dans lequel un personnage n'est créé par personne, ni par un dieu, ni par sa mère, ni par les rois, et qui interroge la question de l'autocréation. Mais pour mieux me saisir de ce conte, il me fallait apprendre le malgache - la langue et la culture malgaches. Au fur et 
à mesure, les événements de 1947 se sont imposés à moi, dans la mesure où ils constituent un moment majeur de destruction identitaire et culturelle. Parler de 1947 s'est imposé au bout de la troisième version de Nour, 1947. Ce livre a cinq versions, je l'ai écrit sur dix ans. Dans la première version, que j'ai donnée à Pierre Astier ${ }^{7}$ en 1994, quand je suis arrivé en France, il n'était pas encore question de 1947. J'ai beaucoup discuté avec lui sur ce premier manuscrit. On est tombé d'accord pour penser que ce n'était pas un livre encore achevé. J'ai refait une deuxième version. Pierre Astier hésitait encore. Finalement il était prêt à le publier, mais c'est moi qui me suis cette fois ravisé. Mon intérêt pour les événements de 1947 est apparu à ce moment-là. Je ne voulais pas commettre d'erreurs sur le plan historique, même si j'écrivais une fiction. Le but n'était pas de faire un livre d'histoire, mais il était important pour moi de mener un travail d'historien.

\section{G. B. : Madagascar, 1947, en revanche, c'est comme un livre d'histoire?}

J.-L. R. : Non, pour moi ce n'est pas un livre d'histoire. Entre Nour, 1947 et Madagascar, 1947, j'ai écrit les nouvelles de Rêves sous le linceul'. Dans ce recueil, il y a cette nouvelle qui s'appelle «Fahavalo » et il y a aussi ces images d'enfants qui se jettent des falaises. En général, les critiques pensent que j'ai puisé ces passages de Nour, 1947 dans Rêves sous le linceul. Mais c'est le contraire : j'ai pris des extraits de Nour, 1947, qui était encore à l'état de brouillon, pour en faire des nouvelles dans Rêves sous le linceul. Je n'arrivais pas à trouver la forme de Nour, 1947. Par exemple, ça paraît évident de dire qu'on va écrire sept nuits, mais ça ne l'était pas pour moi, et je ne l'ai trouvé vraiment qu'à la fin, sans avoir conscience que c'était comme les sept jours de la création dans la Bible. Je pensais alors avoir fini le cycle de 1947. Je pensais ne plus y retourner. Mais on ne tourne pas comme ça la page d'un livre publié. Nour, 1947 a eu des répercussions chez les lecteurs, ce qui m'a forcé en quelque sorte à y revenir.

\section{G. B. : Madagascar, 1947 s'est écrit finalement en réponse à des réactions à Nour, 1947 ?}

J.-L. R. : Non, pas directement. Il s'est tout de même passé sept ans entre les deux livres. J'ai publié Nour, 1947 en 2001, et le projet de Madagascar, 1947 se forme en 2007. Ce projet est vraiment né d'un contexte très précis. Il y a d'abord eu la visite de Jacques Chirac à Madagascar en juillet 2005, lors de laquelle il a reconnu que les massacres de 1947 constituaient un épisode intolérable, même s'il a ajouté qu'il s'agissait seulement d'une dérive de la colonisation. Mais ce qui m'a poussé à écrire Madagascar, 1947, c'est surtout la réaction du président malgache, Marc Ravalomanana, qui prétendait que tout cela n'était pas grave. Lui-même était né en 1949 et il estimait qu'il fallait passer à autre chose. Je trouvais au contraire qu'une chance historique n'avait pas été saisie par Madagascar. C'était tout de même la première fois qu'un président français reconnaissait ce qui s'était passé, même s'il le faisait du bout des lèvres et seulement à la fin de son mandat. Madagascar aurait pu saisir cette chance de mettre les choses sur la table. Et toute l'Afrique aurait suivi. Il y a aussi cette fameuse loi sur la colonisation positive, toujours en $2005^{9}$. En 2007, c'est enfin les soixante ans de l'insurrection de 1947. Jutta Hepke, l'éditrice des Éditions Vents d'ailleurs, est venue me voir en décembre 2006. Il fallait faire quelque chose pour cet anniversaire. Au même moment, j'avais également trouvé des photographies qui appartenaient à une amie: les photographies du fonds Charles Ravoajanahary ${ }^{10}$. Je voulais en faire quelque chose. On est tombé d'accord pour publier un livre. Je voulais sortir le livre le 29 mars 2007, à la date anniversaire du soulèvement. J'ai donc eu quinze jours pour écrire le texte, et Jutta Hepke n'en a pas eu davantage pour confectionner le livre avec mon texte et un choix 
de photographies. Concernant le texte, je me suis appuyé sur les documents que j'avais amassés pour l'écriture de Nour, 1947.

\section{Madagascar, 1947}

G. B. : Dans Madagascar, 1947, il y a en effet beaucoup de documents : des photographies donc, mais aussi des témoignages d'individus qui ont vécu les événements de 1947, des analyses savantes que vous reprenez ou que vous critiquez, etc. Et pourtant, vous dites que ce n'est pas un livre d'histoire.

J.-L.R.: C'est un livre qui emporte le lecteur dans l'émotion. Les historiens ne s'intéressent qu'aux faits. Moi, je suis sur le terrain de la mémoire.

G. B. : Ce terrain-là, c'est celui de la première personne du singulier ?

J.-L. R. : Oui, c'est le je qui prend la parole. Je suis convaincu que c'est une approche qui va davantage sensibiliser les gens que celle d'un livre d'histoire. Le livre de Jacques Tronchon ${ }^{11}$ est une somme dans laquelle on apprend bien plus que dans mon livre. Mais je voulais montrer l'importance que 1947 a sur moi, et par-delà, sur les Malgaches ou même sur toute personne qui se sent concernée.

G. B. : Finalement, l'approche littéraire présente l'intérêt de pouvoir toucher un large public autour de la question de la mémoire, question qu'un historien prendrait moins en charge ou de façon différente.

J.-L. R. : De façon différente, oui. On ne peut pas prendre des arguments d'historien pour critiquer ce livre, notamment l'exigence de l'objectivité, le relativisme lié au contexte, etc. Ce n'est absolument pas l'objet du livre. L'objet du livre, c'est la question du corps. Le corps humain a été complètement profané à Madagascar en 1947 : le corps des vivants et le corps des morts. Les Malgaches prennent soin du corps des morts et, en 1947, ils n'ont pas pu le faire. Madagascar, 1947 est un livre tombeau, un livre linceul pour tous ces corps profanés par la répression. C'est un livre qui incite juste à en apprendre davantage. Pour quelqu'un qui veut vraiment savoir ce qui s'est passé en 1947, ce livre-là ne suffit pas.

G. B. : J'ai lu dans un journal ${ }^{12}$ qu'en mars de cette année 2009, un colloque d'historiens devrait avoir lieu à Antananarivo sur les événements de 1947.

J.-L. R. : Non, non. Le journaliste a mal interprété mon propos. Quand il y a eu la censure de 47, je me suis dit qu'il fallait trouver une réponse, au-delà de l'indignation, pour placer les événements de 1947 au cœur du débat public. Le problème, ce n'est pas la censure ou la liberté d'expression. Après divers projets, j'ai finalement lancé la proposition d'une exposition, qui sera commandée à Pierrot Men, un grand photographe malgache. Cette exposition, portée par la région Île-de-France, sera exposée à Avignon cet été et sans doute en Île-de-France à l'automne.

\section{G. B. : Et à Madagascar?}

J.-L. R. : On avait des contacts avec la mairie d'Antananarivo. Mais comme la mairie est entrée en conflit avec l'État malgache ${ }^{13}$, le projet est ajourné. De plus, le président malgache ne veut pas entendre parler de 1947. Mais on ne désespère pas.

G. B.: Quel rapport entretenez-vous avec les historiens français ou malgaches qui s'intéressent à cette période de l'histoire franco-malgache?

J.-L. R. : Le responsable des recherches sur les événements de 1947 est un universitaire français : Jean Fremigacci. Il est membre d'un centre de recherches à Madagascar et en 
France $^{14}$. On ne peut pas dire que nous abordons cette question de la même manière, notamment dans cette polémique absurde des chiffres portant sur le nombre des morts.

G. B. : Et les chercheurs malgaches?

J.-L. R. : Ils travaillent sous sa responsabilité.

T. B. : Mais les chercheurs malgaches sont en train de se libérer de sa tutelle.

J.-L. R. : Il y a des historiens qui travaillent avec lui mais qui n'ont pas tout à fait la même approche, comme la chercheuse malgache Lucile Rabearimanana ${ }^{15}$. Et je suis beaucoup plus proche d'elle que de lui.

\section{G. B. : Et sur quoi porte le conflit qui vous oppose à Jean Fremigacci ?}

J.-L.R. : C'est à propos des chiffres ${ }^{16}$ mais également de l'approche générale des événements de 1947. Ce qui est étonnant, par exemple, c'est qu'il y a eu très peu d'études conduites à partir des témoignages français et surtout malgaches, alors que des recueils de témoignages ont été constitués. Considère-t-on ces témoignages comme étant dénués de valeur parce que relevant d'une prétendue rumeur, hors de la vérité historique donc? Mais les rumeurs ne trahissent-elles pas les peurs de l'époque, ne sont-elles pas de véritables outils de propagande ou de manipulation de la part des uns et des autres? Et dans ces rumeurs, quelle est la part de vérité ? Un historien est censé travailler sur des archives, certes, mais les témoignages sont aussi des outils parfaitement classiques, ce qui ne semble pas être le cas quand il s'agit des Malgaches. N'a-t-on pas entendu dire que certaines archives étaient fermées, car elles pouvaient mettre en cause des personnalités encore vivantes? De fait, certains témoignages deviennent irrecevables.

G. B. : Par exemple toi, Thierry, même si tu n'es ni malgache ni historien, tu as été sensible à ces témoignages.

T. B. : J'ai rencontré des gens qui m'ont raconté leurs combats, des personnes d'une noblesse incroyable et qui portent cette histoire dans une trop grande solitude. C'est une douleur terrible. J'en ai rencontré quatre, enfin cinq, mais le cinquième n'était plus en état de parler. Ces gens ont entre quatre-vingts et quatre-vingt-dix ans, mais ils sont très clairs. Il est très facile de les croiser à Antananarivo, ce n'est pas la peine d'aller dans les campagnes, mais aucun historien n'est jamais venu les interroger. Ces personnes ont pourtant des choses à dire, et elles ont besoin de les dire.

J.-L. R. : Autre exemple : aucun historien n'exploite les archives de Jacques Tronchon, qui sont des archives absolument fabuleuses, alors qu'elles sont entreposées à cinq cents mètres de l'université à Antananarivo.

T. B. : Jacques Tronchon est un père franciscain qui est historien. Mais personne ne vient le voir. Dans son livre, il écrit qu'il ne veut pas donner ses sources. Jean Fremigacci en a profité pour mettre en cause son chiffrage en expliquant qu'il ne s'appuyait pas sur des sources véritables ${ }^{17}$. Mais Jacques Tronchon a en fait des sources écrites, classées, et même des documents confidentiels qui lui ont été transmis par des officiers français dont il protège le nom. Et tout cela est consultable. Je l'ai fait.

G. B. : On est dans la problématique des conflits de mémoire et, plus précisément encore, dans celle de l'occultation d'une mémoire au profit d'une autre. Ce qui me frappe dans Madagascar, 1947, c'est au contraire la polyphonie des voix qui prennent la parole et se font entendre. Vous m'avez dit, Jean-Luc Raharimanana, que vous aviez écrit Za en pensant que des hommes de théâtre pourraient en faire quelque chose. Lisant Madagascar, 1947, je me 
suis fait la même remarque. Le livre présente en effet dans son dispositif même un grand nombre de voix: voix d'un je, mais aussi lettre d'un ami, propos d'un combattant qui rend visite à votre père, voix savantes des historiens ou des sociologues, voix des hommes politiques, etc. Pourquoi faire entendre toutes ces voix?

J.-L. R. : À Madagascar, les événements de 1947 sont désignés par le terme de tabataba, qui signifie voix, au sens de troubles, clameurs, rumeurs, scandales, désordres, émeutes, colères, etc. Le livre rend compte de cela. Mais je n'ai pas choisi de donner au livre le titre de Tabataba, parce qu'un film de Raymond Rajaonarivelo porte déjà ce titre ${ }^{18}$.

G. B. : Tabataba aurait peut-être été moins clair, en tous les cas pour le lectorat français.

J.-L. R. : Oui, ç'aurait été moins clair. Madagascar, 1947 est un titre beaucoup plus parlant, qui a un côté coup de poing. Mais qui suis-je pour prendre ce titre ? Déjà, pour Nour, 1947, j'hésitais à mettre la date de 1947. Et là, je rajoute Madagascar... Mais du point de vue de la sensibilisation du lectorat, le titre est bien choisi.

G. B. : Concernant cette polyphonie des voix, j'ai une question qui peut paraître annexe mais qui me semble importante et qui touche à la présence des voix féminines dans votre $œ u v r e$. Dans Za aussi, on peut lire les interludes du «Chant de la femme » qui reviennent régulièrement. À la fin de Madagascar, 1947, le je se «réinvente ${ }^{19}$ » en une femme prénommée Antara: pourquoi ce passage d'un sexe à l'autre qui, précisément, fait échapper le livre au genre de l'essai historique?

J.-L. R. : Oui, on passe à la question de savoir comment raconter l'histoire. Le prénom Antara vient d'abord de tantara, qui signifie histoire en malgache. Il vient ensuite d' Antara, qui signifie temps des abysses en malgache. Antara est une déesse aux cheveux d'ombre souvent représentée comme la femme d'un tyran cruel ou d'une bête féroce qui vit au fond de l'océan. Lorsque les hommes (qui sont ses enfants) souffrent, elle sort de l'eau pour effacer leurs souffrances avec ses cheveux. J'avais l'impression que la nation malgache procède de la même façon : effacer l'histoire et reprendre les vagues du présent.

G. B. : Antara est une figure ambivalente. D'un côté, elle efface la souffrance, ce qui est plutôt positif, mais, d'un autre côté, elle le fait d'une telle manière que cette souffrance revient toujours.

J.-L. R. : Oui, elle ne regarde pas en face la douleur de ses enfants. Ce qui m'a intrigué, c'est que cette figure a complètement disparu du corpus des contes malgaches. On la trouve très rarement. C'est aussi la raison pour laquelle je voulais la mettre en avant.

G. B. : Cette figure participe de la polyphonie qui interroge en profondeur les identités sociales et les jeux de pouvoir mais aussi l'altérité interne au sujet lui-même.

J.-L. R. : Ce n'est pas faux. Lorsqu'on parle d'histoire à Madagascar, on a deux versions : la version française et un certain discours malgache parfois manichéen et sans nuance. Sur ce point, je peux rejoindre Jean Fremigacci. C'est une imposture intellectuelle de faire comme si tous les Malgaches avaient été hostiles à la colonisation française. Ceux qui portent aujourd'hui ce discours anti-français sont d'ailleurs les mêmes qui, à l'époque, soutenaient les Français. Les voix qui se sont levées en 1947 et lors des insurrections précédentes ont au contraire été occultées : c'étaient des voix de paysans et de descendants d'esclaves. Je voulais faire entendre ces voix. C'est la raison pour laquelle je n'ai inséré presque aucune voix d'hommes politiques dans Nour, 1947. Les personnages sont des rebelles dans la forêt, qui ne savent pas pourquoi la France occupe le pays et qui ont juste un rapport très profond avec leur terre. Mais il est vrai, par ailleurs, qu'il y a beaucoup de voix en moi. On parle beaucoup à Madagascar. À la maison, il y avait tout le temps des gens qui passaient et qui parlaient. Mais ce sont des 
voix qui n'arrivent jamais à la surface. Cette multiplicité des voix intérieures est aussi liée à une manière d'écrire, puisque, lorsque j'écris, j'efface complètement le je. Je fais comme si je n'existais pas pour que les autres prennent ma place.

G. B. : Finalement, le titre Madagascar, 1947, même s'il y a un je, vous pouvez en assumer la grande généralité.

J.-L. R. : Oui.

\section{De Madagascar, 1947 à 47 : une adaptation pour la scène}

\section{G. B. : Et dans ton travail d'adaptation, Thierry, j'imagine que la multiplicité des voix est centrale.}

T. B. : C'est la multiplicité des voix qui créait le possible théâtral. D'un côté, il y avait le je qui suscitait une figure et, d'un autre côté, ces autres voix qu'on pouvait traiter comme des commentaires. On pouvait monter le texte très directement. Il n'y a eu qu'une seule coupe franche: le passage à propos des tirailleurs sénégalais et de la critique d'Octave Mannoni ${ }^{20}$. Cela surchargeait ce qui déjà était dense. Les autres coupes sont des coupes de plateau, des coupes d'un verbe passant de l'écrit à la scène et qui doit être resserré.

G. B. : Comment avez-vous travaillé à partir du texte ? Vous avez travaillé ensemble sur les coupes? Jean-Luc Raharimanana, vous avez laissé Thierry libre de sa démarche?

J.-L. R. : Oui. Il me demandait ce que j'en pensais et j'approuvais, sans vérifier si ça tenait ou pas.

T. B. : C'est même l'inverse. C'est moi qui lui ai demandé de retraduire son texte en malgache!

J.-L. R. : C'est ça aussi qui était intéressant.

T. B. : Dans 47, il y a de longs passages dits par un acteur malgache en malgache. On joue de cette langue sur le plateau. Il y a un jeu émotionnel sur les langues et sur l'intraduisible.

J.-L. R. : Mais l'autre personnage traduit quand même un peu pour le public.

T. B. : Il y avait un seul souci dans le passage de Madagascar, 1947 à 47, c'était la fin de l'ouvrage, parce qu'elle était plus militante, ce qui me gênait ${ }^{21}$. Jean-Luc l'a resserré pour moi à quelques lignes. Il y avait trop de colère dans ce passage. Mon but à moi, c'était de rendre hommage aux insurgés, de rendre hommage à ces morts. De mon côté, le côté français, il me semblait que je me devais de rendre hommage à ces morts. Ça peut paraître étrange, mais... De la même manière qu'on ne m'a pas appris cette histoire, qui fait tout de même partie de l'histoire de France, puisque Madagascar à l'époque faisait partie de la France, on ne m'a pas appris à respecter ces 40000 à 60000 morts.

J.-L. R. : Le fait de traduire le livre en malgache, ça allait aussi dans ce sens-là : rendre hommage à ces morts. Il s'agissait de donner une langue à ces événements. C'était donc la moindre des choses que le livre soit accessible en malgache. Madagascar, 1947 est maintenant traduit en malgache et publié dans une édition bilingue. 


\section{G. B. : Le travail préparatoire a eu lieu entièrement à Madagascar?}

T. B. : Oui.

G. B. : Il y a la polyphonie des voix dans le livre de Jean-Luc Raharimanana, mais il y a aussi un grand nombre de photographies, on l'a déjà souligné. Tu en as fait un usage pour ta mise en scène?

T.B.: J'ai fouillé moi-même dans des archives et j'ai choisi de projeter une photographie qui montre des gens qui sortent de la forêt sur la route d'Anosibe ${ }^{22}$. J'ai travaillé à partir d'un fond blanc sur lequel, à un moment précis, ces gens apparaissent et sortent de l'image comme des fantômes avec la même stature que les acteurs qui sont devant eux.

J.-L. R. : Les photos du fonds Charles Ravoajanahary sont très abîmées. C'est l'armée française qui les a prises en 1947. En même temps que les bombes, les Français lâchaient aussi des photos pour terroriser les populations et les pousser à sortir de la forêt et à se rendre.

T. B.: Au début du spectacle, on distribue également un journal et, au cours du spectacle, à un moment précis, on donne encore un autre document - comme un acte mémoriel. Ce journal et ces documents comportent des textes écrits de Jean-Luc, de Lucile Rabearimanana, de Césaire ou de Gracq, mais aussi des photographies.

J.-L. R. : Il y a par exemple des photographies de ce que l'on appelait des camps de soumis.

T. B. : J'ai retrouvé les originaux dans une agence photographique à Antananarivo. J'ai retrouvé l'intégralité d'un reportage sur un camp de soumis et je l'ai scanné. Ces images ne sont jamais utilisées par les historiens, comme cette photographie d'août 1948 montrant le colonel Pénard, qui dirigeait la répression dans tout le nord-est de l'île. On voit qu'il a l'air en forme et souriant avec toute son équipe. Pourtant, on sait qu'il avait des soucis à ce moment-là (il l'écrit), parce que les Français cherchaient à rafler tout le monde et que ça résistait encore. Si on regarde bien l'image, on se rend compte que la photo a été prise le même jour et sur la même route que celle montrant les gens qui sortent de la forêt. La plupart de ces archives militaires doivent être entreposées à Vincennes, au Service historique de la Défense.

G. B. : Il y a également dans ta mise en scène un travail sur la matière sonore. Tu peux en dire quelques mots?

T. B. : On entend dans le spectacle la voix des insurgés qui racontent leur combat dans le texte de Jean-Luc. J'ai également travaillé sur des archives sonores de 1947, des informations de l'époque en français.

J.-L. R. : On entend au cours du spectacle la voix de Monja Jaona, grande figure de l'histoire malgache qui a toujours lutté pour la justice, que ce soit en 1947 ou au moment des indépendances, et dont la voix est immédiatement reconnaissable pour les habitants de l'île ${ }^{23}$. Il y a aussi le mot de passe des rebelles, vorona, qui signifie oiseau, qu'on entend une fois et que ceux qui connaissent bien les événements de 1947 identifient là encore tout de suite. Et il y a la voix de Mama Sana, qui est une grande chanteuse traditionnelle ${ }^{24}$. Je l'écoutais avant de connaître Thierry, mais c'est avec son travail que je me suis rendu compte que pratiquement toutes ses chansons évoquent 1947.

T. B. : Mama Sana est présente dans toutes les anthologies qui répertorient les manières les plus inouïes de chanter, en particulier de chanter des épopées. M’intéressant à la 
musique, je connaissais ses interprétations avant de me rendre à Madagascar. Mama Sana s'accompagne d'un simple instrument avec des cordes de fer et, quand la voix part, c'est comme les premiers enregistrements de blues, ça vient d'on ne sait pas où : $\mathrm{du}$ fond du fond de l'origine. J'étais fasciné par cette musique. Je ne connaissais que deux chansons d'elle, et il se trouve que celle que je trouvais la plus extraordinaire traite des événements de 1947, ce que je ne pouvais pas savoir, puisqu'il y avait l'obstacle de la langue. C'est celle qu'on entend dans le spectacle.

J.-L.R.: Mama Sana mélange plusieurs accents de la langue malgache. Elle a une manière de chanter si particulière qu'il faut bien connaître le malgache de sa région d'origine pour la comprendre. Ce n'est pas vraiment le malgache que je connais. Il faut faire un effort. Je savais juste que c'était des épopées. Je l'ai écoutée davantage, j'ai fait un travail de transcription - ce que personne n'avait jamais fait. Et c'est là que j'ai découvert avec stupeur le caractère central des événements de 1947 chez elle.

T. B. : C'est une musique de transe qui vient de très loin. Tu l'écoutes, tu deviens fou.

J.-L. R. : Une fois, j'avais mes nièces à la maison, il y a quatre ou cinq ans. Je passe Marna Sana, et elles ont commencé à trembler. Elles avaient peur... La chanson qu'on entend dans le spectacle est compréhensible par tous. Ce qui est vraiment intéressant, c'est qu'à un moment donné, les paroles disent: "Tsy adala ny malagasy", soit "Les Malgaches ne sont pas fous ", ce qui signifie qu'ils savent très bien ce que les Français ont fait, même si ces derniers prétendent que c'est dans le but de nous civiliser...

G. B. : Le dispositif scénique complexe de 47 vise quel but? Dans un entretien avec JeanPierre Han, tu revendiques, Thierry, d'abord l'idée de donner « une leçon d'histoire », dotée même d'une "morale », puis tu expliques avoir la volonté de faire "un théâtre [...] loin de tout militantisme ${ }^{25}$ ? Comment concilier ces deux impératifs qui peuvent paraître contradictoires, d'autant plus que, comme tu viens de me l'apprendre, tu as supprimé dans 47 les dernières pages de Madagascar, 1947 que tu trouvais trop démonstratives?

T. B. : 47 est une leçon d'histoire, mais je le dis avec un peu d'ironie. C'est quand même étonnant que ce soit des artistes, réputés incohérents et contradictoires, qui se chargent de cela. Si tu veux savoir ce qui s'est passé en 1947 à Madagascar, tu n'as presque rien. D'où l'intérêt de cette leçon au public sur ces événements jamais racontés.

G. B. : Et le terme de «morale»? Tu dégages la «morale» de l'histoire, l'histoire a une « morale » immanente?

T. B. : C'est une deuxième ironie. Mais il ne faut pas oublier de rendre hommage aux insurgés, d'une part, et, d'autre part, il ne faudrait pas oublier non plus de s'insurger un peu plus souvent.

G. B. : Quelle différence entre un théâtre qui pousse à l'insurrection et un théâtre militant ? Que serait un théâtre militant?

T. B. : Un théâtre militant donnerait des réponses. Madagascar, 1947 travaille notre conscience. C'est un texte politique. Comment en est-on arrivé à une telle injustice ? Les mécanismes de la colonisation à Madagascar témoignent d'une forme de folie humaine. 35000 colons contraignent tout un peuple à vivre selon un ordre qu'ils imposent. C'est insupportable, a fortiori en 1947, date à laquelle la nation française se refonde sur l'idéal de la Résistance après avoir éliminé la barbarie nazie. Le monde politique français - et il ne faut pas oublier que ce sont alors deux socialistes qui sont président du Conseil et ministre de l'Outre- $\mathrm{Mer}^{26}-$, va traiter cette insurrection d'une manière radicale. On ne discute pas et on explique qu'il n'y a personne avec qui le faire, alors qu'il y a encore 
peu les Anglais, présents sur l'île de 1942 à la sortie de la guerre, discutaient intelligemment avec les mouvements démocratiques malgaches.

G. B. : Là, tu donnes des réponses, non? Tu rétablis la réalité des faits? En revanche, je trouve intéressant le fait de dire que le texte de Jean-Luc Raharimanana est politique mais pas militant.

T. B. : Il est politique au sens noble du terme. Pour retrouver du politique, ma démarche est de questionner les artistes. J'ai le sentiment que c'est seulement dans les œuvres très fortes que l'on retrouve la grande question politique - celle du comment vivre ensemble. Je n'ai pas l'impression que les hommes politiques, en France ou en Afrique, s'intéressent aujourd'hui à cette question. Cette approche ne conduit pas à un discours militant. Je n'ai pas de leçon à donner à quiconque, et surtout pas aux Malgaches. Mais il y a des questions qui m'animent, comme tout un chacun.

G. B. : La différence avec une démarche militante, ce serait finalement que tu n'arrives pas vers les textes avec quelque chose à dire, qui serait déjà établi et solidifié. Tu élabores, tu proposes quelque chose, vous le faites ensemble, avec une prise de risque.

T. B. : Ce qui n'empêche pas par ailleurs, comme citoyen, d'être engagé dans une action militante. C'est une autre affaire.

\section{Représentations de 47 à Madagascar et en France}

G. B. : La création du spectacle a eu lieu le 19 septembre 2008 au centre culturel AlbertCamus d'Antananarivo. II y a eu une autre représentation le 20. Comment se sont passées ces deux représentations?

T. B. : Au centre culturel Albert-Camus se trouve le seul théâtre en ordre de marche de Madagascar. Ce centre culturel est un lieu d'une grande liberté, surtout en ce moment sous la direction de Bérénice Gulmann, qui est très attentive aux artistes. Il n'est pas le centre culturel des Français. Il fonctionne sur le mode de l'échange et de la confrontation.

J.-L. R. : C'est la raison pour laquelle la création a été possible.

T. B. : C'est aussi la raison pour laquelle elle a posé problème! Bérénice connaît bien l'œuvre de Jean-Luc, elle connaît aussi mon travail. Quand je lui ai expliqué ce projet autour de 1947, on a presque tout de suite pensé que ça pouvait être conflictuel. L'ambassade de France n'a pas brillé par ses encouragements! Mais Bérénice a pensé la même chose que nous: qu'un Français et un Malgache reviennent ensemble sur le drame de 1947, avec en plus un acteur français et un acteur malgache, constitue une réponse qui devrait être donnée tous les jours à des questions que les politiques ne posent pas et que les historiens peinent à envisager. On n'est pas un modèle. Mais il est évident que Français et Malgaches doivent reconsidérer ensemble les événements du passé. En France, nous avons un gouvernement, et il y a même tout un monde intellectuel, qui ne veut pas revenir sur la colonisation. La Guadeloupe en ce moment constitue un exemple parfait des désastres qu'entraîne ce refus ${ }^{27}$.

\section{G. B. : Qui était présent dans la salle, je veux dire : quels types de spectateurs ?}

J.-L.R.: C'est bien qu'il y ait eu des problèmes avant le spectacle: les canaux publicitaires habituels nous ont été fermés. Par exemple, il a été impossible de faire la promotion de 47 à la télévision. Il y avait juste l'affiche devant le Centre. Cela a obligé le Centre culturel à engager des démarches inédites en direction des universitaires et des 
étudiants et à proposer une tarification accessible. Un lien s'est aussi créé avec Lucile Rabearimanana, avec Jacques Tronchon. Si tel n'avait pas été le cas, on aurait eu le public habituel du Centre culturel : un public privilégié de lycéens du lycée français, de Malgaches aisés et d'intellectuels malgaches. Ce public était présent. Mais il y avait aussi un autre public. Les réactions des lycéens et des étudiants m'ont beaucoup intéressé. Ils étaient complètement sous le choc. Je me souviens notamment d'une jeune fille qui me disait que le 29 mars avait toujours été pour elle un jour béni, parce que férié, mais qu'à présent, elle avait pleinement conscience que cette date évoque avant tout des morts ${ }^{28}$. Elle le savait déjà plus ou moins, mais pas à ce point-là.

\section{G. B. : Il y avait combien de spectateurs?}

T. B. : Quatre cents spectateurs les deux soirs, ce qui fait un total de huit cents. La salle était comble.

J.-L. R. : Les anciens rebelles ont réagi aussi. Thierry était déjà allé les rencontrer en juillet 2008. Je suis à mon tour allé les voir pour leur parler de Madagascar, 1947 et pour les inviter au spectacle 47. Ils ont dit oui, mais ils ne sont pas venus (c'est très malgache). Il faut souligner qu'ils n'ont jamais parlé des événements de 1947 ni à un historien ni à un Français. Ce que je n'ai pas bien appréhendé et que j'ai compris par la suite, c'est que pour eux c'était trop tôt pour venir dans un lieu français. Il aurait fallu qu'on aille davantage vers eux. En même temps, et ça m'a beaucoup touché, ils étaient très contents que ça ait lieu. Ils m'ont dit que nous devions revenir, parce qu'ils allaient bientôt mourir et qu'ils avaient encore beaucoup de choses à raconter. J'ai l'impression que la pièce a quelque peu libéré la parole sur 1947. S’il y a un blocage officiel, entre Malgaches, on en a toujours parlé. Mais le rendre public sur une scène, c'est encore autre chose.

T. B. : Ce sont des gens qui ont énormément souffert et qui vivent pour la plupart dans une grande misère. Je me suis senti assez proche assez vite de ces gens. J'y passerais bien beaucoup de temps. Mais l'endroit est très douloureux, parce que...

\section{G. B. : L'endroit ?}

J.-L.R.: Il y a un camp militaire dans lequel les rebelles de 1947 qui vivent à Antananarivo occupent un bureau et se revoient chaque semaine. Ce sont eux qui vont distribuer les pensions militaires des anciens combattants de 1947. Il faut bien distinguer les anciens combattants qui se sont battus pour la France, en 1939-1945, en Indochine ou en Algérie, et les anciens combattants de 1947. Les pensions de la part de la France sont très faibles; les pensions malgaches, encore plus dérisoires. Les rebelles de 1947, pour une grande part, n'étaient pas des militaires de carrière, d'où cette difficulté à les reconnaître comme anciens combattants.

T. B. : Il y a 15000 soldats malgaches qui ont combattu pour la France en 1939-1945.

G. B. : Des représentations ont eu lieu en France, les 26 et 27 septembre 2008 au festival des Francophonies de Limoges. J'aimerais vous poser les mêmes questions : quels types de spectateurs assistèrent aux représentations et quelles furent leurs réactions?

T. B. : Il y a aussi eu des représentations à Cavaillon, à Annecy et à Blois. Les gens ne veulent ou ne peuvent pas parler après le spectacle. Ils sont dans la stupeur. Même si ça reste du théatre, le spectateur est convoqué à prendre en charge les 60000 morts. Ça dépasse le côté malgache et français. 
J.-L.R.: Ce que les gens reçoivent, c'est quelque chose qui les dépasse. Intellectuellement, ils peuvent dire que ce qui s'est passé en 1947 est injuste. Mais d'un point de vue émotionnel, c'est trop fort. On est dans une incarnation qui dépasse là encore la leçon d'histoire. On se rend compte que les rebelles de 1947 étaient des êtres de chair qui ont bel et bien existé.

\section{La polémique, et après...}

G. B. : J'en viens pour finir à la polémique. Je rappelle d'abord les faits. Le 15 novembre 2008, vous écrivez une lettre commune à Bernard Kouchner, ministre des Affaires étrangères, pour dénoncer la «censure d'État», plus précisément la censure de l'État français, puisque 47, pourtant soutenu par Culturesfrance, avait été retiré de la programmation proposée aux centres culturels de la région à la demande de la Direction générale de la coopération internationale et du développement (DGCID). La conséquence est grave, puisque cela signifie que 47 ne pourra pas tourner - comme il était initialement prévu - dans l'océan Indien et en Afrique orientale au printemps et en septembre 2009.

T. B. : J'ai écrit une lettre technique, cosignée par Jean-Luc et Jutta Hepke, pour demander les raisons de ce retrait qui, de fait, met un terme à la diffusion de 47 dans la région. Et Jean-Luc a écrit "Silence sur 47 ", qui est plus brutal et que publie le site Rue89 ${ }^{29}$. Les centres culturels français ont un intérêt économique à choisir un seul et même spectacle, qui va tourner dans l'ensemble des centres. Une réunion de programmation a eu lieu à Addis-Abeba, le 5 novembre 2008, qui a réuni les douze représentants des plus gros centres français sur les vingt et un que comptent l'Afrique orientale et l'océan Indien. 47 était présenté par le Centre culturel Albert-Camus, avec un avis favorable de Culturesfrance, ce qui veut dire que $25 \%$ du budget des représentations était déjà financé. Mais le matin même de la réunion, le spectacle a été retiré par la DGCID des propositions faites aux représentants qui étaient présents à Addis-Abeba.

G. B. : Comme l'indique Libération dans son édition du 22 décembre 2008, Bernard Kouchner vous répond dans une lettre qu'il n'y a pas eu «censure d'État » mais que le spectacle a été retiré pour des motifs purement «budgétaires ». Rien n'empêcherait donc les centres culturels et les Alliances françaises de le programmer.

T. B. : Il ment, dans le sens où ce spectacle n'a pas été présenté à cette réunion de programmation. Il a donc été purement et simplement censuré, puisqu'il ne peut plus circuler dans le réseau des institutions françaises de la région. D'autre part, le ministère lui-même n'a rien à voir avec le financement de ces spectacles, puisque ce n'est pas lui qui les gère. Il est curieux de voir Bernard Kouchner invoquer des motifs budgétaires qui ne dépendent pas de son administration.

J.-L. R. : Le représentant du Centre culturel Albert-Camus, qui était à Addis-Abeba, n'a même pas eu le droit de défendre la pièce.

G. B. : Le spectacle ne sera donc pas diffusé dans la région?

J.-L. R. : Il sera diffusé à La Réunion, probablement dans trois théâtres, ce qui est tout de même intéressant, parce qu'il y a beaucoup de Réunionnais qui, d'une manière ou d'une autre, ont participé à la colonisation malgache. C'est un député réunionnais ${ }^{30}$ qui a proposé devant le parlement français en 1894 de voter la colonisation de l'île. Des colons réunionnais ont été armés par l'armée française en 1947 et ils ont participé à la guerre contre les rebelles. Il y a eu aussi une tentative d'implantation de colons 
réunionnais après l'insurrection. Les "petits Blancs » de La Réunion qui avaient perdu leurs terres au profit des grandes exploitations pensaient ainsi se rabattre sur Madagascar. Mais l'État français s'est retourné contre eux et a privilégié les intérêts de la métropole. La colonisation réunionnaise de Madagascar a été désastreuse pour ces «petits Blancs». En même temps, beaucoup de Réunionnais ont des origines malgaches, et la répression de 1947 a été pour eux aussi un fait d'histoire complètement intolérable. Faire représenter la pièce à La Réunion va donc être passionnant.

G. B. : Et par rapport à la censure du spectacle, quel écho médiatique y a-t-il eu à Madagascar et en France?

T. B. : Le journal L'Express de Madagascar nous a donné la parole. En France, cet épisode a été suivi assez solidement, entre autres par Libération et Le Monde. C'est nous qui avons arrêté au bout d'un moment de répondre aux sollicitations, parce que nous voulions recentrer l'attention, non pas sur cette histoire de censure, mais sur les événements de 1947. Il y a en France une véritable peur autour de ces questions mémorielles. Le fait qu'on enterre une deuxième fois les insurgés malgaches met à jour l'état général de notre société. Mais on ne s'en sortira pas comme ça. On voit chaque jour que le débat revient. L'important, c'était de rebondir autrement. 47 va être rejoué à Avignon le 17 juillet 2009 dans le festival «Contre-courant». Toujours à Avignon, Jean-Luc va lire le 18 juillet des extraits de L'Arbre anthropophage et on va présenter une exposition des photographies de Pierrot Men autour des événements de 1947. Enfin, dans le festival "in ", nous serons présents avec une pièce que j'ai commandée à Jean-Luc et qui s'intitule Les Cauchemars du gecko. C'est un état du monde vu de Madagascar, du Sud, tout à fait désespéré mais salutaire. La pièce sera créée le 20 juillet et elle sera jouée jusqu'au 26 juillet. Elle tournera en France à l'automne. C'est important, parce que c'est un auteur et un metteur en scène invités en même temps, un Français et un Malgache.

\section{NOTES}

1. Voir Jean-Louis Joubert, "Nouvelles écritures dans l'océan Indien », dans Kumari R. Issur et Vinesh Y. Hookoomsing (dir.), L'Océan Indien dans les littératures francophones. Pays réels, pays rêvés, pays révélés, Actes du colloque de juillet 1997 organisé par l'université de Maurice, Paris-Réduit (île Maurice), Karthala / Presses de l'université de Maurice, 2001, p. 113-121; du même auteur, « Madagascar 1947 », dans Véronique Bonnet (dir.), Conflits de mémoire, Paris, Éditions Karthala, 2004, p. 353-361.

2. Les nouvelles de Lucarne ont été publiées en 1996 au Serpent à plumes. Elles ont été reprises dans la collection « Motifs » de la même maison en 1999.

3. L'Arbre anthropophage a été publié aux Éditions Joëlle Losfeld en 2004.

4. La Bibliothèque Censurée était une "revue parlée " animée par Thierry Bedard depuis 2000 en soutien et en hommage au Parlement international des écrivains (créé en 1993). C'était un programme d'essais, d'entretiens, de correspondances, de dialogues d'auteurs ou de textes de fictions qui, mis en scène, questionnaient le monde au risque du langage. Le Parlement international des écrivains et sa revue Autodafé se sont effacés en 2003 au profit du Réseau international des villes-refuges. 
5. Za a finalement été publié aux Éditions Philippe Rey en 2008.

6. Ce titre est aussi celui du texte qui ouvre le roman $Z a$.

7. Pierre Astier était alors éditeur au Serpent à plumes, maison d'édition spécialisée dans la littérature contemporaine internationale qu'il avait fondée en 1993 avec Claude Tarrène.

8. Rêves sous le linceul a été publié en 1998 au Serpent à plumes. Le livre reparaît en 2004 dans la collection « Motifs » désormais propriété du Groupe Privat/Le Rocher.

9. La promulgation le 23 février 2005 d'une loi relative à la « reconnaissance de la nation » et à la «contribution nationale en faveur des Français rapatriés » dont l'article 4 stipule entre autres que « les programmes scolaires reconnaissent [...] le rôle positif de la présence française outremer, notamment en Afrique du Nord" passa d'abord presque inaperçue avant de faire grand bruit. Choqués autant par la défense de la colonisation que par l'empiétement du législateur sur les compétences des enseignants chargés d'élaborer les manuels scolaires, quelques historiens (Claude Liauzu, Gilbert Meynier, Gérard Noiriel, Frédéric Régent, Trinh Van Thao et Lucette Valensi), bientôt rejoints par beaucoup d'autres, s'élevèrent contre la tentative d'imposer une interprétation officielle à un pan de l'histoire de France. Du côté des politiques de droite comme de gauche, le silence fut en revanche dans un premier temps fort troublant. Il fallut attendre les réactions intéressées du président algérien Bouteflika pendant l'été, les émeutes dans les banlieues et le refus très clair d'Aimé Césaire de rencontrer Nicolas Sarkozy à l'automne pour que la question de l'héritage colonial de la France soit remise au cœur de l'actualité. Jacques Chirac décida finalement de saisir le Conseil constitutionnel et, le 16 février 2006, un décret du ministère de l'Éducation nationale abrogea l'alinéa le plus contestable de l'article 4.

10. Un certain nombre des photographies du fonds Charles Ravoajanahary sont reproduites dans le livre de Jean-Luc Raharimanana (voir Raharimanana, Madagascar, 1947, La Roque-d'Anthéon, Éditions Vents d'ailleurs, 2007, p. 40-62).

11. Voir Jacques Tronchon, L'Insurrection malgache de 1947. Essai d'interprétation historique, ParisFianarantsoa (Madagascar), Karthala-Ambozontany, 1986.

12. Voir René Solis et Marie Christine Vernay, « Le cas 47 », Libération, 11 décembre 2008.

13. Jean-Luc Raharimanana fait allusion au conflit qui, depuis le mois de décembre 2008, oppose le président de la république de Madagascar, Marc Ravalomanana, au maire d'Antananarivo, Andry Rajoelina, qui a pris le pouvoir en mars 2009 en devenant le président de la Haute Autorité de transition.

14. Membre du Centre d'études des mondes africains (CEMAf), Jean Fremigacci est aujourd'hui maître de conférences retraité de l'université Paris I. Il a enseigné de 1969 à 1988 à l'université d'Antananarivo.

15. Lucile Rabearimanana est professeur d'histoire à l'université d'Antananarivo.

16. Jean Fremigacci avance le chiffre de 10000 morts pour les massacres perpétrés par l'armée française. Les 20000 ou 30000 autres disparitions auraient été causées d'après lui par la malnutrition et la maladie. Jacques Tronchon avance quant à lui des chiffres beaucoup plus élevés (voir Raharimanana, Madagascar, 1947, op. cit., p. 19-20).

17. Voir en particulier Jean Fremigacci, « 1947 : l'insurrection à Madagascar ", Marianne, ${ }^{\circ}$ 401, 25 au 31 décembre 2004. Et voir aussi le texte écrit par Jean-Luc Raharimanana entre septembre 2005 et juillet 2006, « Madagascar : 29 mars 1947 : des chiffres et des mots autour d'un massacre colonial » à l'adresse http://survie.org/billets-d-afrique/2005/139-septembre-2005/ article/madagascar-29-mars-1947-des (consulté le 27 avril 2009).

18. Tabataba est un film réalisé en 1986 par Raymond Rajaonarivelo. Il raconte l'histoire dramatique d'un village situé à l'est de Madagascar durant l'insurrection de 1947.

19. Raharimanana, Madagascar, 1947, op. cit., p. 39.

20. Pour ce passage, voir ibid., p. 22-28.

21. Pour ce passage, voir ibid., p. 35-39. 
22. Cette photographie ne figure pas dans Madagascar, 1947. Mais elle appartient à la même série que celle qui figure à la page $60 \mathrm{du}$ livre - avec cette différence toutefois : c'est une photographie en pieds.

23. Monja Jaona (1910-1994) est une grande figure de la vie politique malgache du $\mathrm{xx}^{\mathrm{e}}$ siècle. Emprisonné ou mis en résidence surveillée plusieurs fois (par les Français comme par le pouvoir malgache), il lutta contre la colonisation puis en faveur du respect de la démocratie.

24. Mama Sana, née avec le siècle et morte en 1997, est une chanteuse et une joueuse de valiha (cithare tubulaire, instrument emblématique de Madagascar). Ses chants relatent les chroniques du pays Sakalava Menabe situé à l'ouest du pays.

25. Voir Jean-Pierre Han, « 1947, le silence et l'oubli » [Entretien avec Thierry Bedard (mai 2008)], Frictions, $\mathrm{n}^{\circ}$ 13, «Hommage aux insurgés de Madagascar (1947)», septembre 2008, p. 100-105. Dans le même numéro de la revue, on trouvera la version scénique intégrale de 47 , ainsi qu'un inédit de Raharimanana intitulé « 47, rano, rano ».

26. Membre de la SFIO, Paul Ramadier est président du Conseil à deux reprises, du 22 janvier au 22 octobre, puis du 22 octobre au 19 novembre 1947, soit au moment où éclate l'insurrection malgache. Dans son premier gouvernement, le ministre de la France d'Outre-Mer est Marius Moutet, lui aussi membre de la SFIO. Lors de son second gouvernement, Paul Ramadier est luimême ministre de la France d'Outre-Mer.

27. Thierry Bedard fait allusion aux grèves massives déclenchées par le collectif LKP - Liyannaj Kont Pwofitasyon, soit Union contre l'exploitation outrancière - afin de remédier aux conditions de vie faites aux travailleurs les plus pauvres en Guadeloupe. Les négociations salariales furent des plus difficiles. Les grèves commencées en janvier 2009 aboutirent à un protocole d'accord qui mit officiellement fin à la grève le 5 mars.

28. C'est le 29 mars 1947 que commence l'insurrection du peuple malgache.

29. Voir http://rue89.nouvelobs.com/2008/12/05/madagascar-1947-censure-d-etat-pour-unepiece-de-theatre (consulté le 28 avril 2009).

30. François Césaire de Mahy (1830-1906) est ministre de la Marine et des Colonies en 1887-1888. Il soutient alors activement le projet de colonisation de Madagascar. En 1894, la France vote les crédits nécessaires pour mener une expédition de grande envergure afin d'imposer un protectorat sur l'île.

\section{AUTEUR}

\section{GUILLAUME BRIDET}

Université Paris 13, CENEL 